\title{
Framework for Cloud Usability
}

\author{
Brian Stanton ${ }^{1}$, Mary Theofanos ${ }^{1}$, Karuna P Joshi ${ }^{2}$ \\ ${ }^{1}$ National Institute of Standards and Technology, Gaithersburg, MD, USA \\ \{bstanton, maryt\}@nist.gov \\ ${ }^{2}$ Department of Computer Science and Electrical Engineering \\ University of Maryland Baltimore County \\ Baltimore, MD, USA \\ kjoshi1@umbc.edu
}

\begin{abstract}
Organizations are increasingly adopting cloud-based services to meet their business needs. However, due to the complexity and diversity of cloud systems it is important to evaluate the user experience using within a framework that encompasses the characteristics that define the user experience. In this paper, we propose a cloud usability framework to provide a structure to evaluate the key attributes of the cloud user experience. The framework includes five attributes and 20 elements that characterize this user experience. Generally these describe the consumer's expectations of the cloud. The framework can be foundation for developing usability metrics for organizations interested in measuring the user experience when adopting the cloud.
\end{abstract}

Keywords. Usability • Cloud • Framework •

\section{Introduction}

Cloud computing is a model for enabling ubiquitous, convenient, on demand network access to a shared pool of configurable computing resources (e.g., networks, servers, storage, applications, and services) that can be rapidly provisioned and released with minimal management effort or service provider interaction [1]. Organizations are increasingly adopting cloud based services to address their information technology (IT) needs for software, hardware or network bandwidth [2]. To date, the driving forces behind the cloud have been technological advances and business needs that often outpace the needs of the end-user. In order to better enable ready adoption (acceptance and utilization), users' needs (and their goals) must be incorporated into the process of cloud implementation. Fundamentally, users' needs and goals drive usability.

Various cloud service providers provide different models and deployment types of cloud services and so there is no consistency to the user experience. Moreover, cloud services deployed via the web or mobile device are limited by the capabilities of the browser or mobile 
app interface and so often provide user interfaces that are of lower quality than that provided by traditional client server applications on a desktop platform. It is being increasingly recognized by the cloud user community that there is a need for developing cloud usability standards to ensure consistency among different cloud services purchased from different vendors [3]. Lack of a consistent user experience in an enterprise cloud environment can result in longer time to on-board and application into the enterprise cloud, more training requirements and more technical assistance for the end users. This lack of consistency will affect the actual cost of the application to the organization. If an organization with hundreds of end users can reduce their training requirements by a day, they can improve their organizational productivity and realize cost savings.

The cloud pushes us to examine the user experience beyond the standard desktop computing environment of well established usability principles, guidelines, and metrics. Thus we have developed a framework to provide a structure to evaluate the key attributes of the cloud user experience. The framework driven by user needs identifies five attributes further compartmented into elements and associated measures to ensure that critical usability areas are not overlooked.

\section{Background}

The complexity and diversity of cloud systems has made it difficult to evaluate the user experience using typical usability evaluation techniques.

\subsection{Cloud Standards}

National Institute of Standards and Technology (NIST)'s cloud computing initiative was aimed at standardizing the definition of cloud computing, its various models and deployment strategies. NIST's definition of the cloud mandates five essential characteristics [1] of ondemand self service, broad network access, resource pooling, rapid elasticity, and measured service. The NIST deployment models [1] that include Private, Public, Community, and Hybrid cloud models, do not specifically address the human computer interaction or usability attributes that make up the user experience of a cloud service. 
The NIST cloud model enumerates three service models. Usability elements to consider for each service model are different and depend on the level of control the end user and the consumer organization have over the cloud service. The service models include:

- Software as a Service (SaaS): Provides consumers the use of applications in the cloud. The consumer does not control or manage the applications or the environment in which the application is running. In this model the end user and the consumer organization generally have very limited capability of customizing the user interface of the cloud service. For instance, Google Apps (http://www.google.com/enterprise/ apps/business/) that are being adopted as Enterprise Email applications in many organizations have the same look and feel for all the consumers and limit customization of the user interface to colors and company logos.

- Platform as a Service (PaaS): Provides consumers with the ability to deploy applications created or acquired by the consumer using programming languages, libraries, services, and tools supported by the provider. The consumer does not manage or control the cloud infrastructure, but has control over the deployed applications. In this model, the end user and the consumer organization generally have capability of customizing the user interface of the cloud service. However they are limited by the capability of the platform and cannot use other open source languages, like $\mathrm{C}$ or Java, to develop custom applications. For instance, Salesforce.com provides 'Salesforce1 Platform' application [4] as a PaaS cloud model that allows end users to develop apps on the salesforce platform. However, they are limited to the tools provided by Salesforce on that offering and so might be limited in getting the same user interface as other applications in their enterprise cloud that are purchased from other vendors.

- Infrastructure as a Service (IaaS). Provides consumers with the ability to provision processing, storage, networks, and other fundamental computing resources where the consum- 
er is able to deploy and run arbitrary software, which can include operating systems and applications. The consumer does not manage or control the underlying cloud infrastructure but has control over operating systems, storage, and deployed applications. For instance, Amazon Web Services (http://aws.amazon.com/) provide a IaaS environment to their consumers who can develop personal applications using languages and tools of their choice. This model provides end users with the maximum flexibility of customizing their cloud services and so facilitates development of a consistent user interface. However, IaaS cloud services also require consumer organizations to develop in-house expertise to be able to utilize the benefits of the cloud.

As these cloud computing environment characteristics and components demonstrate decisions about moving to the cloud can be overwhelming to organizations/consumers [5]. Many frameworks and taxonomies have been developed to assist enterprises as they consider the use of the cloud $[6,7,8]$. These frameworks generally focus on technical capabilities and measure business services. For example, Garg et al [6] developed a framework that measures the quality and ranks cloud services. Usability features have only been mentioned in passing, if at all.

\section{Proposed Framework for Cloud Usability}

Any usability framework should start with a good understanding of what usability is. For our purposes, we used the International Organization for Standardization (ISO) definition of usability:

Usability is the extent to which a system, product, or service can be used by specified users to achieve specified goals with effectiveness, efficiency, and satisfaction in a specified context of use. [9] 


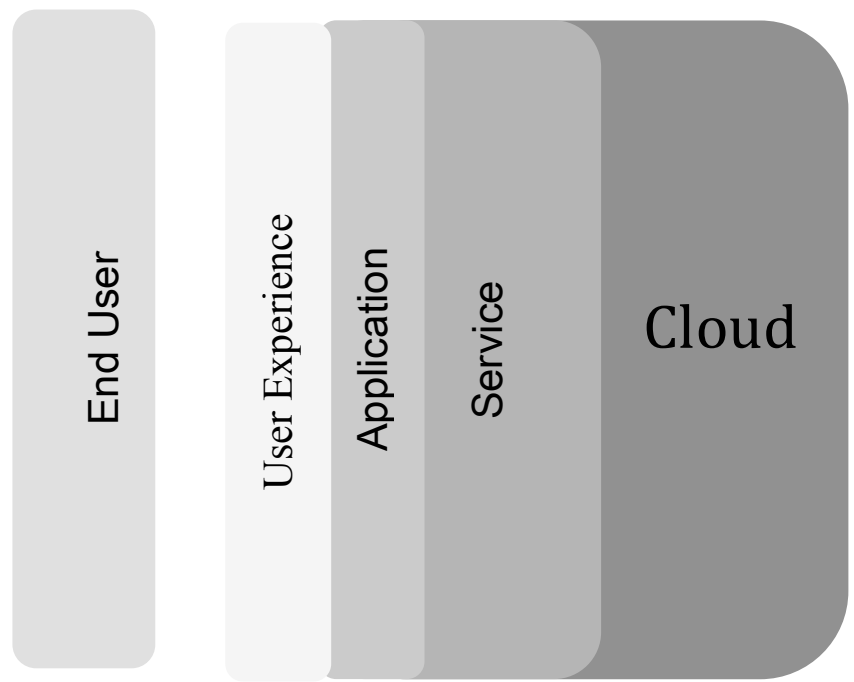

Fig. 1. The cloud user experience

The definition provided us with a roadmap for developing the framework. It first talks about users and their goals that have often been left out when considering the cloud. The definition then goes on to state three metrics: effectiveness, efficiency, and satisfaction. Finally, in the definition, it states that the aforementioned all occur within a specified context of use. It is during the development of the context of use that users' needs (and their goals) are first identified in the User Centered Design (UCD) process [9]. As can be seen in Figure 2 User Needs are central to developing a usable system, product, or service. It is these user needs that form the basis of our framework.

Figure 3 is a pictorial representation of the NIST cloud Usability Framework. This figure lists the various usability attributes that are of interest to cloud users. 


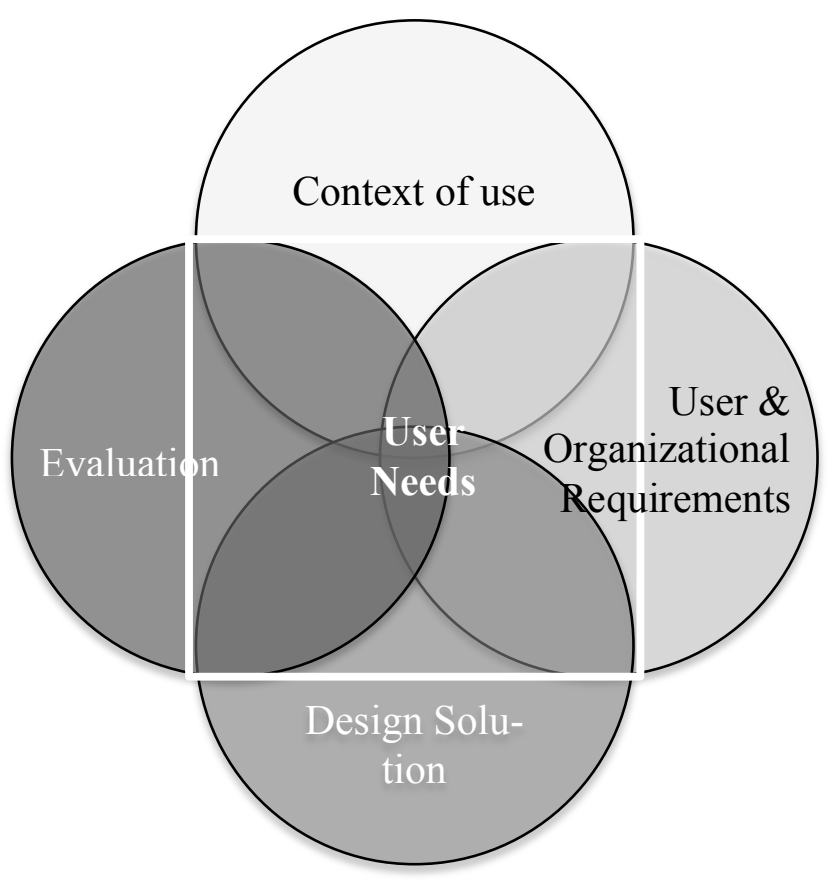

Fig. 2. The User Centered Design process

\subsection{Cloud usability framework}

\begin{tabular}{|c|c|c|c|c|}
\hline \multicolumn{5}{|c|}{$\begin{array}{l}\text { Consumer } \\
\text { (End user } \& \text { organization) } \\
\text { expects the cloud to be: }\end{array}$} \\
\hline Capable & Personal & Reliable & Secure & Valuable \\
\hline Current & Accessibility & Available & Security & Savings \\
\hline Device Independence & $\begin{array}{c}\text { Personalization/ } \\
\text { Customization }\end{array}$ & Responsive & Privacy & User satisfaction \\
\hline Platform Independence & Data Ownership & Consistent & $\begin{array}{l}\text { Identity Management } \\
\text { (authorization) }\end{array}$ & Utility \\
\hline Functions & $\begin{array}{l}\text { Identity Management } \\
\text { (access) }\end{array}$ & Transparency & Trust & \\
\hline & Control & & & \\
\hline
\end{tabular}

Fig. 3. Cloud usability framework 
In a similar vein as the cloud Services Measurement Initiative Consortium (CSMIC) who created a framework to establish measures for quality of cloud services (Service Measurement Index (SMI) [7] we independently created a framework that characterizes the usability attributes that the cloud should contain. The framework's attributes and elements are based on other usability frameworks [10] and ISO usability standards. Consumers should expect the cloud to be Capable, Personal, Reliable, Valuable, and Secure. Figure 3 illustrates this framework.

\section{Capable.}

Cloud consumers should expect their cloud services to have certain capabilities. The four main features that they should look for in a capable cloud service are:

1. Current: The cloud service should be based on the latest technology. The provider should upgrade the service to stay current with the state-of-art of the technology. The service provider should ensure that the cloud service stays compatible with the latest release of hardware, Operating System (OS) and related software applications on which it is dependent. Currently providers upgrade the technology of their services every 18-24 months. In the coming years, we may see a reduction in this time span

2. Platform: Consumers who use IaaS services want to be able to define the exact hardware specifications of the service they wish to purchase. For SaaS services, consumers want the cloud service to be independent of the cloud hardware, Operating System, etc. so that the service can function correctly on any device hardware and software setup.

3. Device: For a cloud service to be capable, consumers should be able to access the cloud service using any device type fixed/mobile etc.

4. Cloud Functionality: cloud services can be considered capable for the consumers if they provide cloud functionality, like elasticity, scalability; rapid provisioning etc., that is not possible in other platforms. 


\section{Personal.}

Cloud Services should allow consumers (organizations and end users) to change the look and feel of the user interface and customize service functionality to suit their needs. At times, an organization's IT policy may not allow the end users to personalize the user interface of any enterprise application; however, they may still customize the basic solution for the whole organization. There are five main elements that a User should expect under the Personal attribute:

1. Accessibility: It is the degree to which a product or system can be used by people with the widest range of characteristics and capabilities to achieve a specified goal in a specified context of use [11]. Cloud services should be accessible to consumers with a variety of needs. For instance, many consumers in the US mandate that the solution should be compliant with the Americans with Disabilities Act (ADA).

2. Customization: Customization is adaptation of a software product to the needs of a particular audience [12]. Cloud services should allow consumers to change their user interface to suit their needs. An organization may choose a standard customized interface for all their end users to ensure lower maintenance needs.

3. Control: The consumer should have a sense of control over the functionality of the cloud service. For instance, they should be able to determine what cookies are set by the cloud service, or be able to switch off GPS tracking if the service is accessed as a mobile application.

4. Data Ownership: Consumers should have ownership over the data they store in the cloud services they use. Users should be able to declare the policies on its usage (in advertisements, other services etc.). For instance, if a consumer uploads pictures to a cloud service, the consumer should be able to determine how that image is used or who has access to it.

5. Identity Management (access): To ensure ease of use, multiple access authentications will have to be implemented in a seamless manner so that the consumer is not aware of the number of authentication/authorization steps they have to go through to access their applications on the cloud. 


\section{Reliable.}

Reliability is the ability of a system or component to perform its required functions under stated conditions for a specified period of time [11]. There are four main elements that cloud consumers look for under the Reliable attribute:

1. Available: Availability is defined as the ability of a service or service component to perform its required function at an agreed instant or over an agreed period of time [13]. Cloud users should expect high availability and it is not unusual to find providers advertising over $99 \%$ availability.

2. Responsive: cloud consumers should want the cloud services to have high degree of performance. The main performance measure that is of interest to the end user is the response time, which is the time it takes the service to respond to a user's request/instruction.

3. Consistent: A cloud service should exhibit the same functionality under every situation. The service should also have no conflicts with the user device type (e.g., different types of desktops) accessing the service.

4. Transparency: The cloud service provider's service policies and technology should be transparent to the cloud consumer organization. Organizational users (like managers, database administrators, etc.) should have access, as needed, to the cloud datacenter and have details about the cloud platform's capabilities and planned changes. This feature is important for building trust between cloud providers and cloud consumers.

\section{Valuable.}

Cloud consumers should expect cloud services to provide value to them and their organization. The three main features that can measure this value include

1. Savings: By using the cloud Service, users will be able to save on the costs it would take to run the same application on their personal infrastructure. In addition they will be able to consolidate and save resources they might need if they ran the same application on their personal infrastructure. 
2. User satisfaction: User satisfaction measures the affective experience of the service. The user satisfaction measure should be high for the user to continue consuming the service.

3. Utility: cloud services will be valuable to the consumers if they can provide new features that are not possible with any other IT setup. For instance, cloud services are proving to be very valuable in -

a. Distributed computing with large datasets (Big Data Applications)

b. Mobile / pervasive computing.

\section{Secure.}

Cloud consumers want their cloud services and its data to be secure.

The three main features that they are looking for in cloud services un-

der this category are

1. Security: The cloud service should be resistant to attacks from unauthorized users, other cloud services, malicious software and attacks on cloud hardware and Internet network.

Security attacks on a service by cloud based attackers compromise the functionality of that Service. While these attacks may not directly harm the user's private data; they will make the cloud service more vulnerable to Privacy attacks.

2. Privacy: The cloud service can prevent leakage of data that compromises end user's private data like personal information, financial accounts, geo-location (if not desired by the user) etc.

Privacy Attacks compromise a cloud user's personal information. These attacks may not directly harm the cloud service's security, but can affect its performance.

To ensure a cloud user's privacy, cloud providers authenticate or validate a user's credentials. Tools, patches, utilities and applications are deployed by the cloud providers to ensure the cloud User's privacy.

3. Identity Management (authorization): The cloud service shouldn't allow unauthorized users to access user data or execute any process. The organization will designate an administrator who will be able to maintain the list of users and their authorization levels 
4. Trust: Cloud consumers should have confidence in the cloud service to, at least, fulfill the conditions set forth in the SLA. Trust is highly correlated with other elements such as privacy, security, and data ownership.

\section{Conclusions and ongoing work}

We have presented a framework for evaluating the user experience of the cloud. The framework builds on techniques, metrics, and guidelines from desktop computing and more recent work in ubiquitous computing. The goal of the cloud usability framework is to provide a structure to ensure that key areas of evaluation and consumer needs are not overlooked.

While this framework is based on evaluations from desktop computing, a literature review and personal experience, we have plans to apply it to a cloud environment.

Finally we believe the framework is the foundation for developing usability metrics for organizations adopting the cloud. Thus our goal is to develop a method of measuring the elements in the framework and defining an associated set of usability metrics.

\section{References.}

1. Mell, P. \& Grance, t. (2011) The NIST Definition of Cloud Computing, (Special Publication 800-145). Gaithersburg MD: National Institute of Standards and Technology.

2. Gartner Predicts Infrastructure Services Will Accelerate cloud Computing Growth (2013, February 19). Retrieved September 12, 2013 from http://www.forbes.com/sites/louiscolumbus/2013/02/19/gartner-predictsinfrastructure-services-will-accelerate-cloud-computing-growth/

3. Singleton D., "It's time for a cloud UI standard", $\mathrm{http}: / / \mathrm{blog}$.softwareadvice.com/articles/enterprise/its-time-for-a-cloud-ui-standard1021412/, last retrieved 18 Nov 2013

4. Salesforce1 Platform, http://www.salesforce.com/platform/overview/, last retrieved 18 Nov 2013

5. Goyal, P., Enterprise Usability of Cloud Computing Environments: Issues and Challenges. 2010 Workshops on Enabling Technologies: Infrastructure for Collaborative Enterprises. (2010), 54-59.

6. Garg, S.K., Versteeg, S., Buyya, R., SMIcloud: A Framework for Comparing and Ranking cloud Services, Utility and cloud Computing (UCC), 2011 Fourth IEEE 
International Conference on, vol., no., pp.210, 218, 5-8 Dec. 2011. doi:

10.1109/UCC.2011.36

7. Cloud Services Measurement Initiative Consortium, The Service Measurement Index. Retrieved September 6, 2013, from http://csmic.org/

8. Vaquero, L.M., Rodero-Merino, L., Caceres, J., and Lindner, M., A break in the clouds: towards a cloud definition. SIGCOMM Comput. Commun. Rev. 39, 1, pp.50-55, Dec 2008, doi: 10.1145/1496091.1496100

9. ISO/IEC 9241 -210:2010 Ergonomics of human-system interaction -- Part 210: Human-centred design for interactive systems

10. Scholtz, J. and Consolvo, S. 2004. Toward a framework for evaluating ubiquitous computing applications. Pervasive Computing, 3, 2, 82-88.

11. ISO/IEC 24765:2010 Systems and software engineering Vocabulary

12. ISO/IEC 26514:2008 Systems and software engineering Requirements for designers and developers of user documentation

13. ISO/IEC 20000-1:2011 Information Technology Service Management 